Kompass

Autoimmun

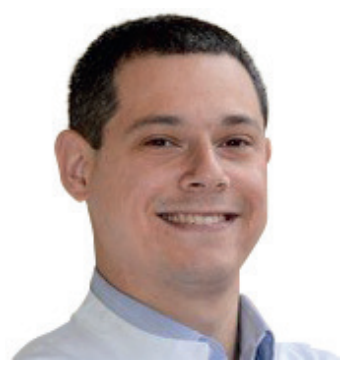

\title{
Prädiktoren für Progression und Mortalität bei Patienten mit RA-assoziierter ILD
}

\author{
Nicolas Carlos Kahn
}

Thoraxklinik Heidelberg, Abteilung für Pneumologie und Beatmungsmedizin, Universitätsklinikum Heidelberg, Heidelberg, Deutschland

Abstract aus Mena-Vázquez N, Rojas-Gimenez M, Romero-Barco CM et al.: Predictors of progression and mortality in patients with prevalent rheumatoid arthritis and interstitial lung disease: a prospective cohort study. J Clin Med 2021;10:874.

\section{Keywords}

Rheumatoid arthritis · Interstitial lung disease · Biologics · Nonanti-TNF biologics

\section{Abstract \\ Objectives: To describe a prospective cohort of patients with rheumatoid arthritis associated with interstitial lung disease (RA- ILD) and identify risk factors associated with disease progression and mortality in this cohort.}

Patients and methods: We performed a multicenter, prospective, observational study of patients with RA-ILD receiving disease-modifying antirheumatic drugs (DMARDs) between 2015 and 2020. The patients were assessed using high-resolution computed tomography and pulmonary function tests at baseline and at 60 months. The main endpoint was «Progression to ILD at the end of follow-up» in terms of the following outcomes: (1) improvement (i.e., improvement in forced vital capacity (FVC) $\geq 10 \%$ or diffusing capacity of the lungs for carbon monoxide (DLCO) $\geq 15 \%$ and absence of radiological progression); (2) nonprogression (stabilization or improvement in FVC $\leq 10 \%$ or diffusing capacity of the lungs for carbon monoxide (DLCO) $<15 \%$ and absence of radiological progression); (3) progression (worsening of FVC $>10 \%$ or DLCO >15\% and radiological progression); or (4) death. We recorded demographic and clinical characteristics, lung function, and the incidence of adverse events. A Cox regression analysis was performed to identify factors associated with the worsening of ILD.

Results: After 60 months, lung disease had stabilized in $66 \mathrm{pa}-$ tients (56.9\%), improved in 9 (7.8\%), and worsened in 23 (19.8\%). Eighteen patients (15.5\%) died, with a mean survival of 71.8 (1.9) months after diagnosis of ILD. The Cox multivariate analysis revealed the independent predictors of worsening of RA-ILD to be usual interstitial pneumonia (hazard ratio (HR), $2.6(95 \% \mathrm{Cl}, 1.0-$ 6.7)), FVC <80\% (HR, $3.8(95 \% \mathrm{Cl}, 1.5-6.7))$, anticitrullinated protein antibody titers (HR, $2.8(95 \% \mathrm{Cl}, 1.1-6.8))$, smoking $(\mathrm{HR}, 2.5(95 \% \mathrm{Cl}$, 1.1-6.2)), and treatment with abatacept, tocilizumab, or rituximab $(\mathrm{HR}, 0.4(95 \% \mathrm{Cl}, 0.2-0.8))$. During follow-up, 79 patients (68\%) experienced an adverse event, mostly infection (61\%). Infection was fatal in 10/18 patients (55.5\%) during follow-up.

Conclusions: Lung function is stable in most patients with RAILD receiving treatment with disease-modifying anti-rheumatic drugs (DMARDs), although one-third worsened or died. Identifying factors associated with worsening in RA-ILD is important for clinical management. 


\section{Transfer in die Praxis}

\section{Hintergrund}

Eine interstitielle Lungenerkrankung (ILD) ist die häufigste pulmonale Manifestationsform der rheumatoiden Arthritis (RA) und kommt in 10-15\% der Patienten mit RA vor, wobei diese Angabe möglicherweise eine Unterschätzung ist, da bei Patienten mit RAILD bislang keine standardisierten oder regelmäßigen ScreeningUntersuchungen auf das Vorliegen von ILD stattfinden. In aktuellen Studien werden Prävalenzen einer ILD bei Patienten mit RA im CT-Thorax-Scan (hochauflösende Computertomografie (HRCT)) von $27-67 \%$ bei meist asymptomatischen Patienten berichtet [1] Das Vorliegen einer RA-ILD ist mit einer schlechteren Prognose und erhöhten Mortalität für die Betroffenen verbunden. In Beobachtungsstudien konnte ebenfalls gezeigt werden, dass eine Subgruppe von Patienten mit RA-ILD einen ähnlichen Verlauf aufzeigt wie Patienten, die an einer idiopathischen Lungenfibrose (IPF) leiden [2]. Die IPF ist eine Form der progredient verlaufenden ILD, die radiomorphologisch und histologisch durch ein UIP-Muster (usual interstitial pneumonia) charakterisiert ist. Dieses Muster lässt sich auch in einer Subgruppe von Patienten mit RA-ILD nachweisen.

Die hier diskutierte Studie hatte sich zum Ziel gesetzt, Risikofaktoren für die Prognose und Mortalität bei Patienten mit RA-ILD zu identifizieren

\section{Ergebnisse der Studie}

Diese prospektive, multizentrische Beobachtungsstudie aus Spanien, an der 6 Zentren teilgenommen haben, hat insgesamt 116 Patienten mit einer RA-ILD über 60 Monate nachverfolgt. Die Endpunkte wurden definiert als a) Progression der ILD zum Ende des Followups, b) Stabilisierung oder Verbesserung der Lungenfunktion ohne radiomorphologische Progression der ILD, c) Progression (definiert als Verschlechterung der forcierten Vitalkapazität (FVC) > 10\% oder der Diffusionskapazität (DLCO) > 15\%) oder Tod. Die radiomorphologischen Muster der ILD wurden gemäß der Konsensus-Klassifikation der American Thoracic Society (ATS)/European Respiratory Society (ERS) [3] im Sinne einer NSIP (non-specific interstitial pneumonia), UIP und anderen (Bronchiolitis, organisierende Pneumonie, gemischte Muster) eingeordnet.

Die Patienten waren im Schnitt 68,3 Jahre alt mit etwa gleicher Verteilung der Geschlechter. Alle in die Studien eingeschlossenen Patienten wurden mit DMARD (disease-modifying antirheumatic drugs) behandelt, meist in Kombination mit anderen Medikamenten. Die ILD war im Schnitt bereits seit 2 Jahren bekannt, interessanterweise wurde bei Studieneinschluss nur 1 Patient mit einem antifibrotischen Medikament (Nintedanib) behandelt. Bis zum Ende des Beobachtungszeitraums wurde nur 1 weiterer Patient zusätzlich mit diesem Medikament behandelt.

71 (61\%) der Patienten hatten als radiomorphologische Muster der ILD ein UIP-Muster, 41 (35,4\%) eine Form der NSIP-Muster und die übrigen 4 Patienten ein anderes ILD-typisches Muster. Bei RA-ILD wird das UIP-Muster im Vergleich zu z.B. Kollagenose-assoziierten
ILD vermehrt beobachtet, diese Beobachtung konnte in dieser Studie ebenfalls bestätigt werden.

Nach 60 Monaten Follow-up verblieben noch 98 Patienten (84,5\%) in der Studie. Hiervon wurden 23 (19,8\%) als progredient eingestuft (Verschlechterung der Lungenfunktion) und 18 (15,5\%) waren verstorben. Im HRCT-Scan wurden 32 Patienten (27\%) als progredient eingestuft. Die weitere Analyse der Daten ergab, dass eine Therapie mit Nicht-anti-TNF-bDMARD (biologische DMARD, wie z.B. Abatacept, Rituximab oder Tocilizumab) mit einem 50\% niedrigeren Risiko für ein Voranschreiten der RA-ILD assoziiert war. In dieser Gruppe war auch das Gesamtüberleben signifikant besser im Vergleich zu Patienten mit anderen Therapieregimen (62,7 Monate im Vergleich zu 54,5 Monaten). Das Vorhandensein eines radiomorphologischen UIP-Musters für sich genommen war bereits mit einem 2,5-fach erhöhten Risiko für Progression oder Tod verbunden im Vergleich zu Patienten mit einem NSIP-Muster. Diese Assoziation konnte auch schon in anderen Studien festgestellt werden [4].

Eine Therapie mit Methotrexat (MTX) wurde, im Gegensatz zu der oft im klinischen Alltag inkorrekt gestellten Annahme, nicht mit einem höheren Risiko für eine RA-ILD assoziiert. Aktuelle Studien zeigen, dass zwar in den ersten Monaten einer Therapie mit MTX die Gefahr des Auftretens einer exogen allergischen Alveolitis etwas erhöht ist [5], ein Zusammenhang mit dem vermehrten Auftreten einer RA-ILD besteht allerdings nicht. Ein höheres Risiko für Progression oder Tod hatten Patienten, die zu Beginn der Nachbeobachtungszeit rauchten, ein radiologisches UIP-Muster oder hohe ACPATiter (anti-citrullinierte Protein/Peptid-Antikörper) aufwiesen oder deren FVC bereits zu Beginn < 80\% war.

\section{Fazit für die Praxis}

Die Studie von Mena-Vázquez et al. zeigt erneut, wie relevant das Erkennen einer pulmonalen Manifestation im Sinne einer RA-ILD bei Patienten mit einer RA ist und dass eine Objektivierung des Risikos für einen rasch progredienten Verlauf oder einen frühzeitigeren Tod durch vergleichsweise einfach zu bestimmende Parameter möglich erscheint. Um besser abschätzen zu können, ob ein solcher Verlauf durch den Einsatz unterschiedlicher Therapieregime zu beeinflussen ist, reicht die Anzahl der Patienten in dieser Studie allerdings nicht aus. Es bleibt abzuwarten, ob ein vermehrter Einsatz von antifibrotischen Medikamenten (z.B. Nintedanib) ebenfalls einen relevanten Einfluss auf die Gesamtpopulation der Patienten mit RA-ILD, speziell diejenigen mit einem radiomorphologischen UIP-Muster, haben werden. Die in dieser Richtung ermutigenden Ergebnisse der INBUILD-Studie [6] haben auf diese Studie noch keinen Einfluss nehmen können, da die INBUILD-Studie erst 2019 publiziert wurde.

Es bleibt spannend zu sehen, ob in den kommenden Jahren weitere Studien zeigen können, dass Endpunkte wie Progression und Überleben bei Patienten mit RA-ILD durch diese neuen Erkenntnisse und Modifizierung der Therapieregime signifikant beeinflusst 
werden können. In jedem Fall sollte bei Patienten mit einer RA-ILD eine Mitbetreuung durch ein spezialisiertes ILD-Zentrum angestrebt werden.

\section{Disclosure Statement}

The author has nothing to declare in regard to this publication.

\section{Literatur}

1 Robles-Pérez A, Luburich P, Bolivar S, et al.: A prospective study of lung disease in a cohort of early rheumatoid arthritis patients. Sci Rep 2020;10:15640,

2 Morisset J, Vittinghoff E, Lee BY, et al.: The performance of the GAP model in patients with rheumatoid arthritis associated interstitial lung disease. Respir Med 2017;127:51-56.
3 Travis WD, Costabel U, Hansell DM, et al.: An official American Thoracic Society/European Respiratory Society statement: Update of the international multidisciplinary classification of the idiopathic interstitial pneumonias. Am J Respir Crit Care Med 2013;188:733-748.

4 Li L, Liu R, Zhang Y, et al.: A retrospective study on the predictive implications of clinical characteristics and therapeutic management in patients with rheumatoid arthritis-associated interstitial lung disease. Clin Rheumatol 2019:39:1457-1470.

5 Conway R, Low C, Coughlan RJ, et al.: Methotrexate and lung disease in rheumatoid arthritis: a meta-analysis of randomized controlled trials. Arthritis Rheumatol 2015;66:803-812.

6 Flaherty KR, Wells AU, Cottin V, et al.: Nintedanib in progressive fibrosing interstitial lung diseases. N Engl J Med 2019;381:1718-1727.

Korrespondenz an:

Dr. Nicolas Carlos Kahn, nicolas.kahn@web.de 